\title{
Theory of Intelligent Act
}

Mehran Spitmaan ${ }^{1^{*}}$, Amanda Caterina Leong ${ }^{2^{*}}$

${ }^{1}$ Department of Psychological and Brain Sciences, Dartmouth College, $\mathrm{NH}$

${ }^{2}$ Interdisciplinary Humanities Program, University of California, Merced, CA

* Equal contribution

Corresponding author: ACL, interdisciplinary Humanities Program, University of California, Merced, CA 95344, aleong11@ucmerced.edu

Manuscript Information: 1 page.

Abstract 199 words

Keywords: intelligence, intelligent act, uncertainty, prediction, characterization

Topics: Consciousness; Critical Thinking;

Disciplines: Artificial Intelligence; Literature; 


\section{Abstract}

Considering the concept of intelligent agents in system theory $(1,2)$, we aim to expand the definitions of intelligence and intelligent act through creating a new theoretical framework and investigating its applications; in order to open new perspectives both in artificial intelligence and literary studies. Assuming a temporally unidirectional environment, our theory describes an intelligent agent as one who aims to perform intelligent acts. The intelligent act is an ongoing attempt made by an agent to predict the future of the self as well as the future of the part or whole of the entire environment. However, the outcome is always uncertain. Our theory categorizes human behavior into intelligent and non-intelligent actions.

Applying this categorization on literary texts enables us to continuously portray characters as intelligent or non-intelligent entities based on their actions. This approach helps redefine the way we perceive agency in characters' progression. By investigating depictions of uncertainties in characters, we consider characters as entities who are able to rupture conventions governing the need for character coherence and narrative closure in literary texts. Therefore, we hope that readers will be able to accept stories that are not only at odds with the characters themselves but also with the readers. 


\section{References}

1. Russell, Stuart J., and Peter Norvig. Artificial intelligence: a modern approach. Malaysia; Pearson Education Limited,, 2016.

2. Franklin, Stan, and Art Graesser. "Is it an Agent, or just a Program?: A Taxonomy for Autonomous Agents." International Workshop on Agent Theories, Architectures, and Languages. Springer, Berlin, Heidelberg, 1996. 